\title{
Percepção do estigma da epilepsia em professores do ensino fundamental
}

\author{
Paula Teixeira Fernandes \\ Elisabete Abib Pedroso de Souza \\ Universidade Estadual de Campinas
}

\begin{abstract}
Resumo
A epilepsia é uma condição neurológica crônica que traz implicações psicológicas e acadêmicas para a criança, sua família, professores e amigos. Este trabalho teve por objetivo identificar a percepção do estigma da epilepsia em professores, levando-se em consideração os aspectos acadêmicos, psicológicos e culturais desta condição. Para isto, foram distribuídos questionários a respeito da epilepsia para professores do ensino fundamental da rede pública de ensino em Campinas. Diante dos 94 questionários respondidos, observou-se que $91,5 \%$ dos professores pesquisados sabem que a epilepsia é uma desordem neurológica; 40,0\% acham que o tratamento deve ser feito com acompanhamento médico e 96,8\% acreditam que as crianças com epilepsia devem freqüentar escola comum. Em relação às crenças, a que mais apareceu foi o medo da criança engolir a língua durante a crise. Os professores pesquisados mostraram possuir conhecimentos adequados a respeito da epilepsia, o que contradiz o observado nas atitudes sociais encontradas em nossa sociedade, que são caracterizadas por preconceito e estigma.

Palavras-chaves: epilepsia infantil; estigma; escola
\end{abstract}

\begin{abstract}
Perception of epilepsy stigma in fundamental school teachers. Epilepsy is a common neurological disorder that brings psychological and academic implications to the children, their family, teachers and friends. The purpose of the study was to identify teachers' perception of epilepsy as a stigma, considering the academic, psychological and cultural aspects of this condition. Questionnaires about their knowledge of epilepsy were distributed to teachers of public schools in Campinas, Brazil. From the 94 answered questionnaires, it was observed that $91.5 \%$ knew that epilepsy is a neurological disorder, $40.0 \%$ thought that the treatment is with medical follow-up, and $96.8 \%$ believed that children must study in a normal school. In relation to beliefs, the fear of a child swallowing the tongue during the seizure appeared in $45.7 \%$ of teachers' answers. The searched teachers showed that they have adequate knowledge about epilepsy. However, this reaction contradicts the social attitudes present in our society, which are characterized by prejudice and stigma.
\end{abstract}

Key-words: childhood epilepsy; stigma; school

A epilepsia é uma condição neurológica crônica muito comum que afeta todas as idades, raças, classes sociais e países. Seu diagnóstico revela dificuldades nas áreas psicológica, física, social e econômica com conseqüentes influências sociais, familiares e escolares, especialmente devido ao desconhecimento sobre a epilepsia, às crenças, ao medo e ao estigma associado (Fernandes \& Souza, 2001; Jallon, 1997; Meinardi, Scott, Reis, \& Sander, 2001). Possui alta prevalência em crianças, o que, de acordo com Lefrève (1980), acontece devido ao fato das crianças possuírem o sistema nervoso em processo de desenvolvimento, estando mais sujeitas a infecções, traumas, constituindo assim, um grupo mais suscetível à epilepsia. Apesar de ser considerada uma condição neurológica, as implicações da epilepsia vão além disso, afetando o comportamento, o ajustamento psicossocial e a qualidade de vida do pacientes e das pessoas envolvidas (Chung, Chang, Lai, \& Lai, 1995; Fernandes \& Souza, 2001; Reynolds, 2001; Rosa, 1997). As dificuldades aparecerem por vários fatores, incluindo as próprias crises, a medicação antiepiléptica, as implicações psicossociais e os problemas de comportamento, além do preconceito e do estigma sentido pelas pessoas com epilepsia (Chung et al.,1995; Kankirawatana, 1999; Trimble \& Dodson,1994).

Os pacientes com epilepsia sofrem com os comportamentos exibidos pela sociedade, pois muitas vezes as crises inesperadas e imprevisíveis assustam quem não conhece o que é epilepsia, especialmente em ambientes sociais, como a escola, no caso das crianças (Cunha, 2002). 
Além disso, muitas pessoas possuem crenças irracionais provenientes, sobretudo, da falta de conhecimento a respeito da epilepsia. Embora alguns saibam que as crises refletem algo anormal no funcionamento da atividade cerebral, mitos e preconceitos sobre causas e conseqüências ainda existem. Alguns pacientes acreditam que a cada crise algumas células do cérebro são destruídas; outros acreditam que existe risco de morte. Para outros pacientes, as crises continuam a representar um tipo de maldição ou aflição espiritual (Baker, Jacoby, Buck, Stalgis, \& Monnet, 1997; Devinsky \& Penry, 1993; Gumnit, 1997).

Por ser uma condição antiga e com imprevisibilidade de crises, a epilepsia sempre possuiu um caráter místico dependente de interferências sobrenaturais, sendo conhecida como o mal sagrado (Silveira, 1998). Por essas razões já enraizadas na história, a epilepsia é uma condição estigmatizante "por excelência”. Por causa de sua incerteza clínica e do seu significado social, o impacto psicológico da epilepsia na vida das pessoas é muito significativo (Baker et al., 1997). Todas essas idéias inadequadas acabam sendo reforçadas culturalmente, promovendo sentimentos e atitudes negativas em relação às pessoas com epilepsia (Fernandes \& Souza, 2001).

No que se refere aos aspectos escolares, também aparecem muitas idéias inadequadas. Sabe-se que a freqüência da epilepsia é alta na idade escolar e muitas crianças acabam não se saindo bem na escola, tendo dificuldades de aprendizagem e de interação social. Estas dificuldades podem estar ligadas não apenas à própria epilepsia (idade de início, tipo de epilepsia, etiologia e tratamento, grau de controle das crises, tipo e dosagem da medicação), mas especialmente a fatores psicológicos, como: qualidade de instrução, baixa expectativa dos professores e pais, baixa auto-estima da criança, rejeição dos professores e colegas, entre outros (Kankirawatana, 1999; Souza \& Guerreiro, 2000).

Diante deste contexto, é importante a avaliação dos conhecimentos dos professores do ensino fundamental para que, a partir desta, exista uma divulgação de conhecimentos adequados sobre a epilepsia, no sentido de se criar condições favoráveis em todos os aspectos da vida: familiar, social, profissional ou escolar (Rosa, 1997), para que se consiga uma mudança de atitude em relação às crianças com epilepsia.

O fato de se conhecer as idéias e comportamentos dos professores permite uma medida parcial do que a comunidade escolar pensa sobre a epilepsia.

Assim, o objetivo do presente trabalho foi identificar a percepção dos professores do ensino fundamental a respeito do estigma na epilepsia, levando-se em consideração os aspectos acadêmicos, psicológicos e culturais desta condição.

\section{Método}

\section{Participantes}

Professores do ensino fundamental (primeiro grau) de quatro escolas da rede pública de ensino da cidade de Campinas.

\section{Instrumento}

O questionário proposto foi elaborado a partir de temas levantados na literatura nas pesquisas de qualidade de vida e estigma (Fernandes \& Souza, 1999; Fernandes \& Souza, 2001; Guerreiro et al.,1994; Souza et al.,1998) e experiência clínica. Este questionário (ver Apêndice) era auto-aplicativo, com 13 questões fechadas de múltipla escolha e possibilidade de comentários a respeito dos seguintes aspectos:

- conhecimentos gerais a respeito da epilepsia (questões 2, 7 e 9): o que os professores entendem por epilepsia, quais são as possíveis causas e os tratamentos adequados;

- informações sobre a epilepsia (questões 1 e 6): qual o nível de informação dos professores a respeito da doença, como tiveram acesso a estas informações;

- crenças (questão 3): idéias inadequadas a respeito da doença, como por exemplo, é contagiosa, a criança pode engolir a língua, etc.;

- atividades educacionais (questão 8): o que os professores pensam sobre a educação escolar das crianças com epilepsia - devem ou não freqüentar escolas regulares, devem participar de atividades esportivas, etc.; e

- experiências pessoais com epilepsia (questões 4, 5, 10, 11 e 12): o que sentem ao ver uma crise, o que fazem para ajudar, contato com alunos ou outras pessoas com epilepsia.

\section{Procedimento}

Foram distribuídos 140 questionários para professores do ensino fundamental das quatro escolas que participaram da pesquisa. Eles recebiam o questionário e poderiam devolvê-lo no dia seguinte. Não era necessária a identificação dos professores.

\section{Resultados}

Dos 140 questionários distribuídos, 94 foram devolvidos respondidos, o que corresponde a $67,14 \%$.

Os resultados são apresentados de acordo com os diferentes aspectos analisados.

\section{Conhecimentos gerais a respeito da epilepsia}

Os resultados mostram que a maioria dos professores (91,5\%) sabe que a epilepsia é uma desordem neurológica, apenas 3,2\% consideram-na como uma desordem de desenvolvimento e 1,0\% acham que a epilepsia é uma doença mental.

A respeito do tratamento da epilepsia, apareceram como respostas: medicação adequada, acompanhamento médico, cirurgia, acompanhamento psicológico e acompanhamento psiquiátrico, como mostrado na Figura 1.

Como pode ser visto na Tabela 1, a maior parte (65,3\%) dos professores atribui fatores genéticos à epilepsia.

\section{Informações sobre a epilepsia}

A maioria dos professores (64,9\%) considera seu conhecimento sobre a epilepsia regular/suficiente; $11,7 \%$ o consideram bom, 13,8\% o consideram ruim, e 6,4\% o consideram péssimo. $1,0 \%$ dos professores acham que seu conhecimento é ótimo. 


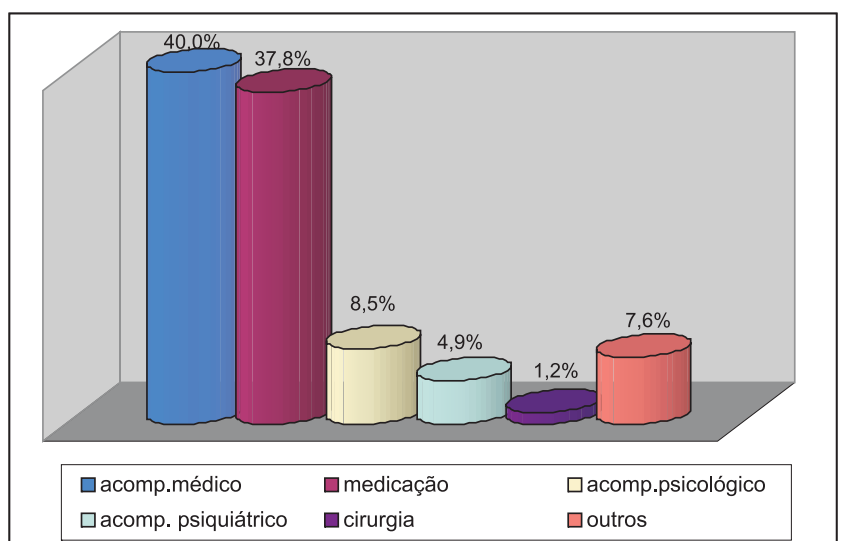

Figura 1. Tratamento da epilepsia, segundo os professores.

Tabela 1

Causas atribuídas à epilepsia pelos professores

\begin{tabular}{lc}
\hline \multicolumn{1}{c}{ Causas da epilepsia } & Freqüência (\%) \\
\hline Fatores genéticos & 65,3 \\
Traumas / agentes físicos & 19,8 \\
Vermes & 3,9 \\
Doenças infecciosas & 1,0 \\
\hline
\end{tabular}

Também a maioria dos professores (58,5\%) já obteve alguma informação sobre a epilepsia. A maior parte destas informações foi obtida através de amigos, como pode ser visto na Tabela 2.

Tabela 2

Origem da informação sobre epilepsia

\begin{tabular}{lc}
\hline $\begin{array}{c}\text { Origem da } \\
\text { informação }\end{array}$ & $\begin{array}{c}\text { Freqüência } \\
(\%)\end{array}$ \\
\hline Amigos & 24,5 \\
Parentes & 16,4 \\
Professores & 13,6 \\
Médicos & 12,7 \\
\hline
\end{tabular}

\section{Crenças}

As crenças que mais apareceram dizem respeito ao medo de a criança engolir ou enrolar a língua durante a crise, medo de que a criança com epilepsia desenvolva uma doença mental futura ou não acompanhe a escola ou o medo de que a epilepsia seja uma doença contagiosa (Figura 2).

Em relação às atividades educacionais, a grande maioria dos respondentes $(96,8 \%)$ acha que as crianças com epilepsia devem freqüentar escola comum, apenas 2,1\% consideram importante o estudo em casa e 1,0\% acham que o ideal é a classe especial.

Em relação às atividades esportivas, 46,0\% dos professores acreditam que as crianças devem participar, com res-

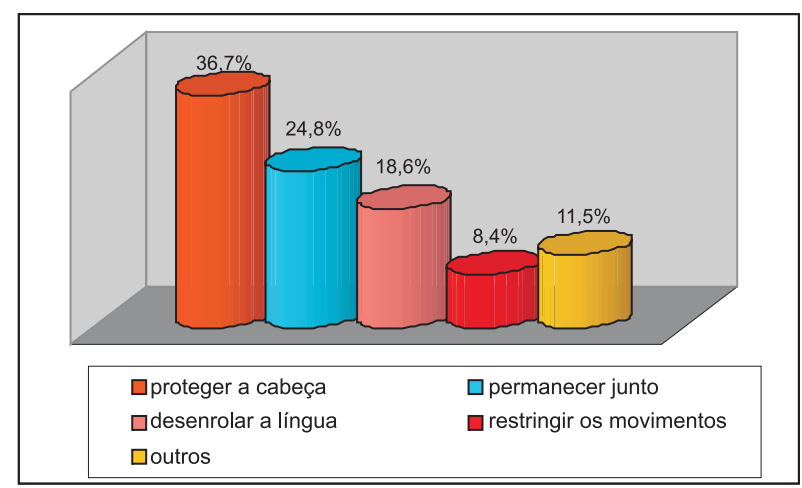

Figura 2. Crenças relativas à epilepsia.

trições, das atividades físicas e esportivas e 43,4\% acham que não é necessário fazer restrições às mesmas.

\section{Experiências pessoais com epilepsia}

Os resultados mostram que, ao ver uma criança tendo uma crise, a maioria dos professores $(66,5 \%)$ relata preocupação (Tabela 3).

Tabela 3

Reações dos professores diante de uma crise epiléptica

\begin{tabular}{lc}
\hline \multicolumn{1}{c}{ Reações } & $\begin{array}{c}\text { Freqüência } \\
(\%)\end{array}$ \\
\hline Ficam preocupados & 66,5 \\
Sentem dó ou pena & 11,0 \\
Sentem medo & 9,3 \\
Não sabem como ajudá-las & 8,5 \\
Rejeitam as crianças & 1,7 \\
\hline
\end{tabular}

Em relação ao que fazer durante uma crise, os professores acharam que é preciso proteger a cabeça da criança, permanecer junto à mesma, desenrolar sua língua, restringir seus movimentos, entre outros (Figura 3).

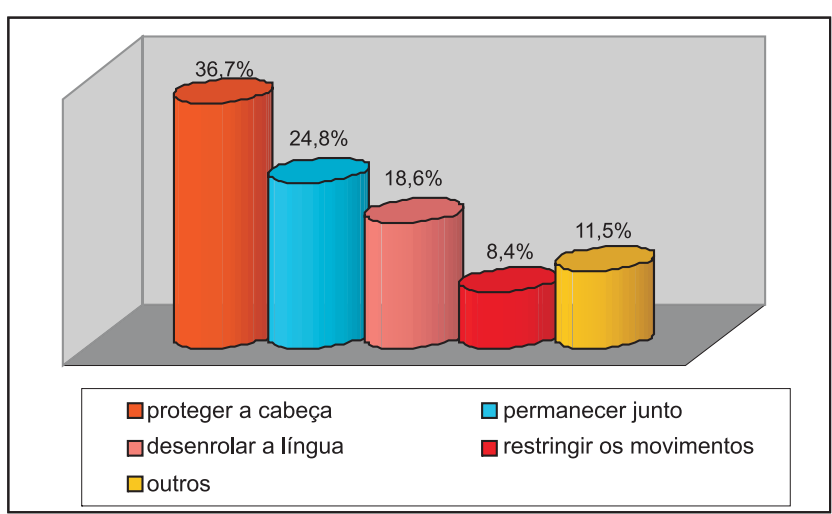

Figura 3. Como proceder durante uma crise epiléptica. 
No que diz respeito ao convívio com pessoas portadoras de epilepsia, 6,4\% dos professores têm alunos portadores de epilepsia, 13,8\% conhecem alguém com epilepsia na escola em que trabalham e 13,8\% possuem familiares com epilepsia.

\section{Discussão}

Por ser uma condição neurológica crônica muito comum na infância, a epilepsia acarreta um profundo impacto na qualidade de vida, influenciando não só o comportamento da criança e seus pais, como também as relações sociais e escolares, trazendo preocupação no que se refere ao futuro das crianças e ao término das crises (Hanai, 1996; Souza \& Guerreiro, 2000).

Neste estudo, foi possível verificar que muitos professores pesquisados demonstraram possuir informações adequadas a respeito da epilepsia. Mas, apesar de 64,9\% dos professores considerarem seus conhecimentos suficientes, existem algumas lacunas nestas informações, com crenças e preconceitos.

No que se refere às causas da epilepsia, a maioria dos professores fala de aspectos importantes e corretos, mas também houve a presença de vermes como causadores da epilepsia, mostrando desconhecimento sobre o assunto.

No que diz respeito às crenças, algumas ainda persistem na área educacional: o medo de que a criança possa engolir a língua durante a crise e o medo da criança com epilepsia apresentar uma doença mental futura.

$\mathrm{O}$ medo de a criança engolir a língua durante a crise epiléptica é, de acordo com alguns autores (Ford, Gibson, \& Dreifuss, 1984), bastante comum e acaba controlando o comportamento dos pais, professores ou responsáveis de colocarem dedos, canetas, réguas dentro da boca da criança para evitar que a língua se enrole. Por esta razão, há a necessidade de orientação sobre como agir no momento da crise. A outra crença que apareceu fala do medo da epilepsia causar uma doença mental no futuro, o que coincide com os dados de Thompson e Upton (1994).

A presença destas crenças, ou seja, idéias irracionais transmitidas sem base científica, decorrentes do desconhecimento sobre a epilepsia e seu tratamento podem gerar o estigma e, com isso, comportamentos inadequados, super proteção e sentimentos de medo, preocupação e insegurança, dificultando assim os relacionamentos sociais, afetivos e acadêmicos da criança (Devinsky \& Penry, 1993; Souza \& Guerreiro, 2000). Isso porque os professores, diante do que ouvem a respeito da epilepsia (crenças vindas do senso-comum), ficam confusos e preocupados na maneira de lidar com seus alunos portadores de epilepsia.

Algumas respostas inadequadas apareceram, como por exemplo, a necessidade de se colocar a criança com epilepsia em classes especiais, que podem ser resultados de experiências individuais do medo da criança apresentar uma crise dentro da sala de aula e os professores não saberem como lidar com esta situação (Kankirawatana, 1999).
Um outro fator importante é que a falta de vivência com pessoas portadoras de epilepsia (apenas 6,4\% dos professores possuem alunos com epilepsia) pode gerar maneiras idealizadas de se lidar com o problema.

Em relação aos aspectos educacionais, os professores, em geral, apresentaram um posicionamento educacional adequado e esperado, provavelmente porque pessoas com maior grau de instrução, como professores, possuem melhores informações e recursos e, por isso, uma atitude mais positiva no lidar com a epilepsia (Mielke, Adamolekun, Ball, \& Mundanda, 1997). No entanto, isso não faz parte das atitudes sociais comuns encontradas, que são caracterizadas por crenças irracionais, estigma, discriminação e preconceito. Uma pesquisa recente mostrou que 86\% dos sujeitos (pais de crianças com epilepsia) possuem crenças a respeito da epilepsia (Fernandes \& Souza, 1999). Isso pode explicar porque muitas crianças encontram barreiras para desenvolver seu potencial na escola que não dizem respeito à doença ou aos efeitos colaterais dos medicamentos, mas sim às atitudes sociais para com elas.

Estudos mostram que alguns tipos de síndromes epilépticas, como a epilepsia benigna da infância, normalmente não comprometem o desempenho escolar das crianças. Nestas crianças, o aparecimento de queixas escolares pode ser explicado, não por déficits intelectuais, mas sim por outras condições sócio-culturais, como a presença de estigma e preconceito relacionado à doença e conseqüentes reações inadequadas de pais e professores. O não saber lidar com a epilepsia pode gerar baixas expectativas nos adultos e baixa auto-estima e baixa auto-confiança nas crianças portadoras de epilepsia (Fernandes \& Souza, 2001; Kankirawatana, 1999; Souza \& Guerreiro, 2000).

O posicionamento educacional adequado e o alto índice de informações dos professores desta pesquisa podem estar relacionados a dois aspectos importantes. O primeiro é o tipo de questionário que foi aplicado: perguntas com respostas fechadas, o que já dava pistas das alternativas corretas. A segunda hipótese levantada é que os professores que devolveram os questionários respondidos, provavelmente são os professores que possuem maior conhecimento sobre a epilepsia. Os que não responderam, são os professores que provavelmente não sabem muito a respeito da epilepsia.

A epilepsia é um peso não só para a criança e sua família, como também para os professores, pois no ambiente escolar podem aparecer problemas relacionados à restrição de atividades esportivas, ao medo da crise ocorrer na frente de outras crianças, ao medo da rejeição por causa da epilepsia, entre outros, que podem gerar dificuldades no lidar com estas crianças no dia-a-dia.

\section{Conclusão}

Este estudo reflete apenas uma parte da atitude social a respeito da epilepsia. Apesar da quantidade de respondentes desta pesquisa ter sido pequena, os professores correspondem a um grupo de profissionais instruídos. Mui- 
tas vezes, eles são "modelos” para as crianças e exercem uma influência significativa sobre as mesmas, devendo por isso, possuir conhecimentos e atitudes adequadas sobre a epilepsia, minimizando os efeitos do estigma associado.

Podemos dizer que o trabalho com professores do ensino fundamental visa a identificação das dificuldades relacionadas à epilepsia para, posteriormente, promover a redução do estigma. Acreditamos que professores mais capacitados melhorem a maneira de lidar e de se relacionar com crianças portadoras de epilepsia, para a construção de uma comunidade bem informada para lidar com as diferenças e com o preconceito relacionados à epilepsia.

\section{Referências}

Baker, G. A., Jacoby, A., Buck, D., Stalgis, C., \& Monnet, D. (1997). Quality of life of people with epilepsy: an European study. Epilepsia, 38(3), 353362.

Chung, M. Y., Chang, Y., Lai, Y. C., \& Lai, C. W. (1995). Survey of public awareness, understanding, and attitudes toward epilepsy in Taiwan. Epilepsia, 36(5), 488-493.

Cunha, R. (2002). Educação especial tenta afastar estigma da epilepsia. Revista Com Ciência, 34, 54-56.

Devinsky, O., \& Penry, J. K. (1993). Quality of life in epilepsy: the clinician’s view. Epilepsia, 34(suppl 4), S4-S7.

Fernandes, P. T., \& Souza, E. A. P. (1999). Inventário simplificado de qualidade de vida em epilepsia infantil: primeiros resultados. Arquivos de Neuropsiquiatria, 57(1), 40-43.

Fernandes, P. T., \& Souza, E. A. P. (2001). Identification of family variables in parent's groups in children with epilepsy. Arquivos de Neuropsiquiatria, 59(4), 854-858.

Ford, C. A., Gibson, P., \& Dreifuss, F. E. (1984). Psychosocial considerations in childhood epilepsy. In F. E. Dreifuss (Org.), Pediatric epiteptology classification and management of seizures in the child (pp. 277-285). Massachussets: John Wright \& Sons

Guerreiro, M. M., Silva, E. A., Scotoni, A. E., \& Souza, E. A. P. (1994). Qualidade de vida em epilepsia na infância. Jornal da Liga Brasileira de Epilepsia, 7(1), 21-26.

Gumnit, R. J. (1997). Living well with epilepsy. Nova York: Demos Vermande. Hanai, T. (1996). Quality of life in children with epilepsy. Epilepsia, 37(suppl 3), 28-32. Jallon, P. (1997). Epilepsy in developing countries: ILAE workshop report. Epilepsia, 38, 1143-1151.

Kankirawatana, P. (1999). Epilepsy awareness among school teachers in Thailand. Epilepsia, 40(4), 497-501.

Lefrève, A. B. (1980). Neurologia infantil: semiologia, clínica e tratamento. São Paulo: Sarvier.

Meinard, H., Scott, R. A., Reis, R., \& Sander, J. W. A. S. (2001). The treatment gap in epilepsia: the current situation and way forwards. Epilepsia, 42, 136-149.

Mielke, J., Adamolekun, B., Ball, D., \& Mundanda, T. (1997). Knowledge and attitudes of teachers towards epilepsy in Zimbabwe. Acta Neurologica Scandinavica, 96, 133-137.

Reynolds, E. H. N. (2001). ILAE/IBE/WHO global campaign "out of the shadows”: global and regional developments. Epilepsia, 42(8), 1094-1100.

Rosa, M. L. R. (1997). Obstáculos percebidos por pais e professores no atendimento das necessidades de criança com epilepsia. Revista Latino-Americana de Enfermagem, 5, 37-44.

Silveira, P. R. (1998). O diagnóstico das epilepsias. Informação Psiquiátrica (Universidade do Estado do Rio de Janeiro), 17(1), 26-32.

Souza, E. A. P., \& Guerreiro, M. M. (2000). Qualidade de vida e epilepsia. In C. A. M. Guerreiro, M. M. Guerreiro, F. Cendes, \& I. Lopes-Cendes (Orgs.), Epilepsia (pp. 223-229). São Paulo: Lemos.

Souza, E. A. P., Nista, C. R., Scotoni A. E., \& Guerreiro, M. M. (1998). Sentimentos e reações de pais de crianças epilépticas. Arquivos de Neuropsiquiatria, 56(1), 39-44.

Thompson, P. J., \& Upton, D. (1994). Quality of life in family members of persons with epilepsy. In R. Trimble \& W. E. Dodson (Orgs.), Epilepsy and quality of life (pp. 19-31). Nova York: Raven.

Paula Teixeira Fernandes, mestre em Ciências Médicas pela Universidade Estadual de Campinas, é psicóloga e doutoranda em Ciências Médicas pela mesma universidade. Endereço para correspondência: Av. Saudade, 549 ap. 144-B; Campinas, SP; CEP 13041-670. Fone/Fax: (19) 3232-2540. E-mail: paulatfb@terra.com.br ou paula@aspebrasil.org

Elisabete Abib Pedroso de Souza, doutora em Ciências Médicas pela Universidade Estadual de Campinas, é professora no departamento de Neurologia, Faculdade de Ciências Médicas, Universidade Estadual de Campinas. 


\section{Apêndice}

\section{Questionário de percepção de estigma para professores do primeiro grau}

Prezados professores,

Gostaríamos de contar com sua colaboração, respondendo as questões deste questionário. Precisamos conhecer o que as pessoas sabem a respeito da epilepsia. Suas respostas nos darão pistas para futuras intervenções na comunidade. Lembre-se que não é necessário identificar-se e algumas perguntas permitem mais de uma alternativa de resposta.

1. Qual o seu conhecimento sobre epilepsia?

$$
\begin{aligned}
& \text { ( ) ótimo } \\
& \text { ( ) bom } \\
& \text { ( ) regular / insuficiente } \\
& \text { ( ) ruim } \\
& \text { ( ) péssimo }
\end{aligned}
$$

2. Você acha que a epilepsia é:

$$
\begin{aligned}
& \text { ( ) desordem neurológica } \\
& \text { ( ) doença mental } \\
& \text { ( ) desordem de desenvolvimento } \\
& \text { ( ) outros: }
\end{aligned}
$$

3. Quais são suas idéias a respeito da epilepsia?

- é uma doença que pega? （ ） sim （ ） não

- a criança que possui epilepsia futuramente vai apresentar uma doença mental? ( ) sim ( ) não

- durante a crise, a criança pode engolir a língua? （ ） sim （ ) não

- as crianças com epilepsia acompanham a escola? （ ） sim （ ） não

4. O que você sente ao ver uma pessoa ter uma crise?

$$
\begin{aligned}
& \text { ( ) medo } \\
& \text { ( ) preocupação } \\
& \text { ( ) rejeição } \\
& \text { ( ) dó } \\
& \text { ( ) outros: }
\end{aligned}
$$

5. O que você acha que poderia fazer para ajudar a criança no momento de crise?

( ) nada

( ) desenrolar a língua

( ) dar algo para cheirar

( ) jogar água na criança

( ) proteger a cabeça para não machucar

( ) permanecer junto à criança

( ) colocar algo na boca

( ) não restringir os movimentos

( ) outros:

6. Você teve acesso a alguma informação sobre epilepsia? ( ) sim （ ） não

Onde?

$$
\begin{aligned}
& \text { ( ) livros. Quais? } \\
& \text { ( ) revistas. Quais? } \\
& \text { ( ) televisão. Qual programa? } \\
& \text { ( ) parentes } \\
& \text { ( ) amigos } \\
& \text { ( ) professores } \\
& \text { ( ) outros: }
\end{aligned}
$$


7. O que você sabe a respeito do tratamento da epilepsia?

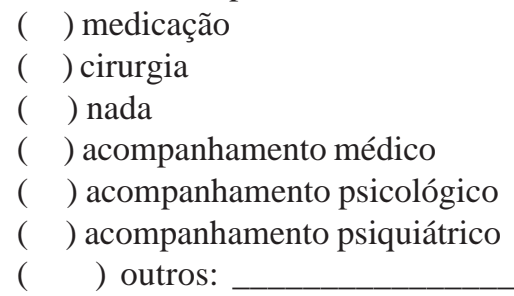

8. O que você pensa sobre a atividade educacional com crianças com epilepsia?

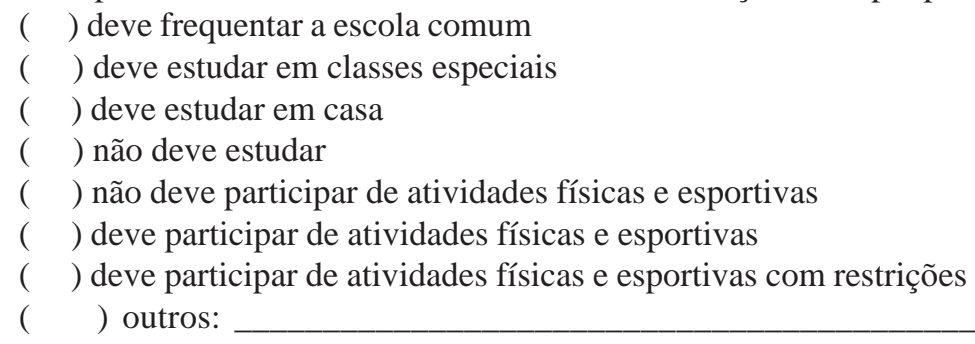

9. Você acredita que a epilepsia seja causada por quais fatores?
( ) verme
( ) fatores tóxicos
( ) mau olhado
( ) fatores genéticos
( ) castigo de Deus
( ) vontade de alguma coisa
( ) doenças infecciosas
( ) traumas ou agentes físicos
( ) outros:

10. Você tem algum aluno ou aluna com epilepsia?

$$
\begin{aligned}
& \text { ( ) sim } \\
& \text { ( ) não }
\end{aligned}
$$

11. E na escola em que você trabalha, conhece alguém com epilepsia?

$$
\begin{aligned}
& \text { ( ) sim } \\
& \text { ( ) não }
\end{aligned}
$$

12. Na sua família, existe alguém com epilepsia?
( ) sim
( ) não
Quem?

13. Diante deste questionário, o seu conceito do que venha a ser epilepsia continua o mesmo?

$$
\begin{aligned}
& \text { ( ) sim } \\
& \text { ( ) não } \\
& \text { Por quê? }
\end{aligned}
$$

